\title{
On the Use of Optimized Monte Carlo Methods for Studying Spin Glasses
}

\author{
E. Marinari ${ }^{1}$, G. Parisi ${ }^{1}$, F. Ricci-Tersenghi ${ }^{2}$ and F. Zuliani ${ }^{1}$ \\ ${ }^{1}$ Dipartimento di Fisica, INFN and INFM, Università di Roma La Sapienza, \\ P. A. Moro 2, 00185 Roma, Italy. \\ 2 The Abdus Salam International Center for Theoretical Physics, Condensed Matter Group, \\ Strada Costiera 11, P.O. Box 586, I-34100 Trieste, Italy.
}

(October 31, 2000)

\begin{abstract}
We start from recently published numerical data by Hatano and Gubernatis [1] to discuss properties of convergence to equilibrium of optimized Monte Carlo methods (bivariate multi canonical and parallel tempering). We show that these data are not thermalized, and they lead to an erroneous physical picture. We shed some light on why the bivariate multi canonical Monte Carlo method can fail.
\end{abstract}

PACS numbers: 75.50.Lk, 75.10.Nr, 75.40.Gb

One of the main problems of numerical results originated from large scale numerical simulations is that checking them is a task that is frequently of the order of magnitude of checking a real experiment: only repeating the full simulation, that demands availability of computer time and codes, allows a full check of the results.

Here we will use as a starting point the work of reference [1] to discuss a few points both about optimized Monte Carlo algorithms and about the behavior of $3 D$ Edwards-Anderson (EA) spin glasses in the low $T$ phase. We will start by showing that the numerical results reported in reference [1] , as far as the low $T$ values are concerned, are wrong: they are not equilibrium averages over the Boltzmann probability. Because of that the physical conclusions reached in the paper, supporting a trivial behavior of the broken phase of $3 D$ spin glasses, are wrong. On the contrary recent numerical simulations [2] support, in this respect, a behavior of the system consistent with the Replica Symmetry Breaking (RSB) picture [3]. We will also shed some light on why the optimized Monte Carlo method used in [1] can fail.

In the following we will first analyze our numerical data obtained by the Parallel Tempering Monte Carlo method [4], focusing on the analysis needed to establish that thermal equilibrium has been reached [5]: we will use a large number of severe criteria that ensure that thermalization has been reached. After showing that the results of [i] are not correct in the low $T$ region we will discuss some preliminary simulations done using the same method used in [1], a bivariate version of the Multi-Canonical Monte Carlo [6], and we will point out a series of reasons for which a non careful implementation of this strategy can fail.

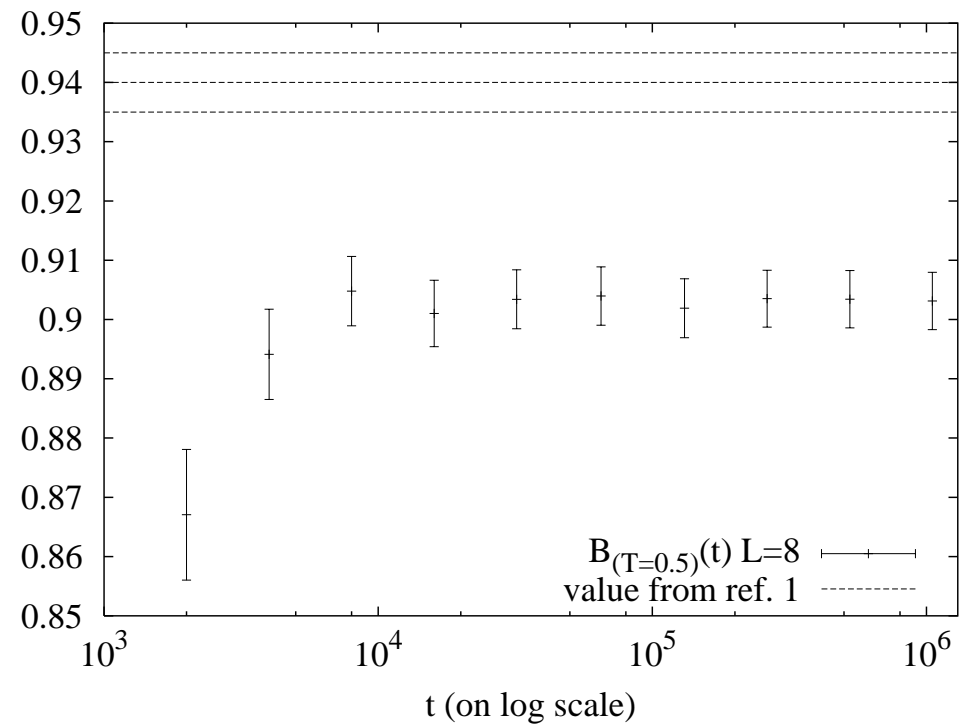

FIG. 1. The Binder parameter, $B(t)$, averaged over logarithmic time windows, as a function of time, at $T=0.5$. 
Let us start from our numerical data obtained through parallel temperingף. We have simulated a $3 D$ EdwardsAnderson spin glass, with binary random quenched couplings, linear size $L=8$ (the largest size used in [1]), down to $T=0.5 \simeq 0.5 T_{c}$ : let us note that in our simulations for the same $T$ values we are able to thermalize reliably lattices up to $L=16$, and that we just discuss here results about the $L=8$ lattice, where we are completely confident about thermalization, only because this is the largest lattice studied in [1]. We use a minimum value of the temperature $T_{\min }=0.5$, a number of temperatures $N_{T}=49$ and a constant temperature step $\delta T=\frac{1}{30}$. The measured correlation times are always smooth functions of $T$ and no anomalies are detected.

Our data at high $T$ turn out to be statistically compatible with the ones of [1]: in the high $T$ region there are no problems.

In figure 11 we plot the value of the Binder parameter,

$$
B(t) \equiv \frac{1}{2}\left(3-\frac{\overline{\left\langle q^{4}(t)\right\rangle}}{\overline{\left\langle q^{2}(t)\right\rangle}}\right),
$$

averaged over logarithmic time windows, as a function of time at $T=0.5$ (close to $0.5 T_{c}$ ). Averaging over logarithmic windows is the safe approach to check convergence in time. We first average over the last half of the total time extent of the run: this is the last point on the right of the plot. We subdivide in the same way the other half of the data, and the second point on the right is the average over the second half of this time span: we continue in this way till the origin of our Monte Carlo run. With a straight line we plot the asymptotic data from [1] as extracted from figure 7 in the paper (since we were estimating by hand we have been generous on the statistical error): here there is no time dependence, we only plot with a straight line the asymptotic value. The discrepancy of our data and the data of [1] is very large and statistically very significant: definitely not an accident.

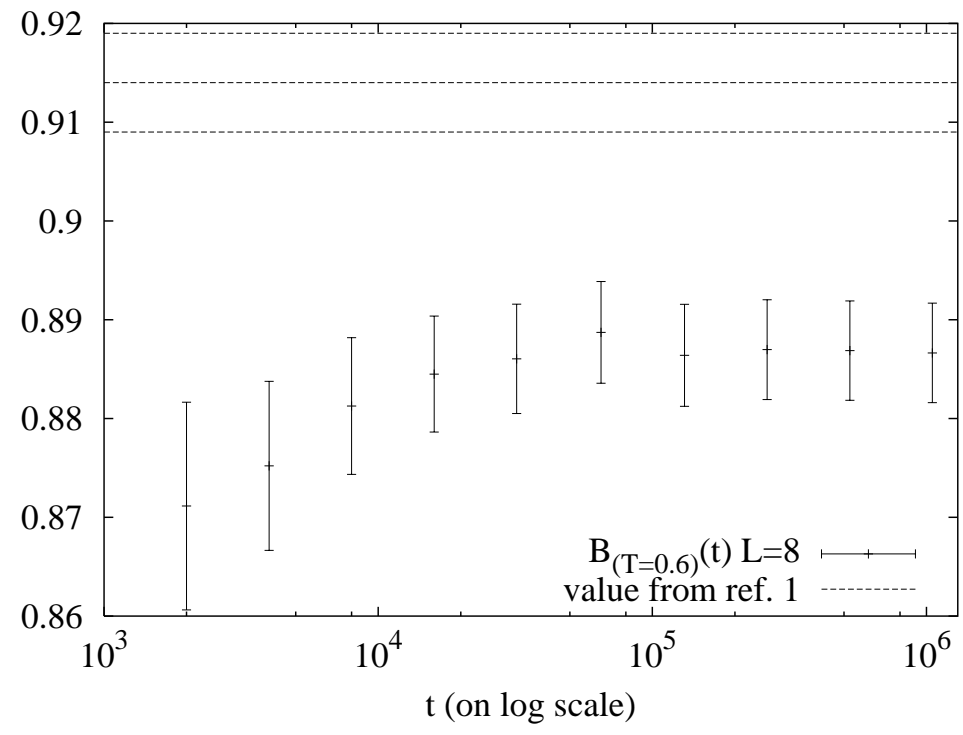

FIG. 2. As in figure 1 , but for $T=0.6$.

In figure 2 we plot the $T=0.6$ data from the same run, always for the Binder parameter averaged over logarithmic time windows: here $T$ is higher, and one could feel safer about thermalization, but again there is a clear and significant discrepancy among our data and the ones of [1]. The dramatic stability of our data for $B(t)$ at low $T$ is already a very good indicator of a high level of thermalization. The results are stable at least during the last eight subdivisions of our two million step runs, i.e. at least from times going from $10^{4}$ to $2 \cdot 10^{6}$.

In order to be sure we are not trapped in some metastable situation we have to check standard criteria about convergence, that in the case of optimized dynamics can be quite difficult to assert [5]. Let us note for example

\footnotetext{
${ }^{1}$ For sake of a complete reliability and without fear of appearing over cautious we have chosen to rewrite all our codes in a double blind pattern, with two different sets of programmers, using different programming languages and different random number generators: they always give statistically compatible results.
} 
that in recent numerical simulations [2] a careful discussion shows that weaker criteria can be sufficient to guarantee thermalization, making in this way possible to simulate more disorder sample with the same amount of computer time (since one needs less thermal sweeps per sample). Here, since thermalization is the main issue, we will check all of the most stringent criteria.

First of all we have checked the acceptance rates of the tempering sweeps in temperature: a bad choice of the $T$ values can make the swap of the temperature value too rare. In our case the rates are very high, of the order of .7 in all the temperature range: our parallel tempering scheme is performing very well.

Secondly we have checked, as customary, if all configurations (we have, as we said, 49 of them) have spent a similar amount of time in each one of the 49 allowed $T$ values. This criterion is important, since the first one could not be sufficient: spin configurations could be spending time swapping among neighboring $T$ values locally, but never leave the high or the low $T$ region. Our permanence histograms are very good: because of the large time extent of the runs all configurations have visited all regions of the $T$ phase space, and the permanence histograms are very flat. Again, this is a powerful test of thermalization.

The last point we have checked is the symmetry under the exchange $q \rightarrow-q$ of the $P_{J}(q)$ for the individual samples. Since the overall flip of all spins is supposed to be a very slow mode of the dynamics, once we have good statistics on this mode we expect to have reached all the relevant regions of the phase space. Again, the symmetry is excellent for all individual samples (even for the more complex samples where the $P_{J}(q)$ has a non-trivial structure).

We consider this body of evidence as clear: our data are thermalized, the numerical data hint evidence in favor of the RSB picture (as confirmed by the data of [2], where even at very low $T$ values one sees that $P(0)$ does not depend on $L$ ) and the method used in [1] did not allow a proper thermalization.

In order to get a better understanding of the situation, and some hints about the reason of the failure of [1] we have implemented a code for rerunning their bivariate multi-canonical simulations.

Our simulations closely follows the description given in the Appendix of reference [1] and by Hatano himself [7]. The analysis of few samples of sizes $L=4,6,8$ has been sufficient in order to understand where the thermalization problems may come from. Unless differently specified we have always used $10^{6}$ Monte Carlo Sweeps (MCS) for thermalizing and $10^{7} \mathrm{MCS}$ for taking measurements in each multi-canonical cycle. The same number of MCS has been used by the authors of [1] only for $L=10$ [7] (less iterations have been used for smaller lattice sizes).

The most delicate point during the thermalization process is the role played by the entropic barriers during the multi-canonical simulation. In a model which undergoes a first order transition the slowing down of the simulation at the critical point is essentially due to the presence of a huge energetic barrier between the two free energy minima. In this case the multi-canonical simulation works fine [6], and it rapidly converges towards a regime where every energy is sampled with the right probability, i.e. uniformly. Problems may arise when the multi-canonical method is applied to spin glasses or in general to models where entropic barriers play a central role. To this respect the study of its performances in models with only entropic barriers (e.g. backgammon model [8]) would be illuminating.

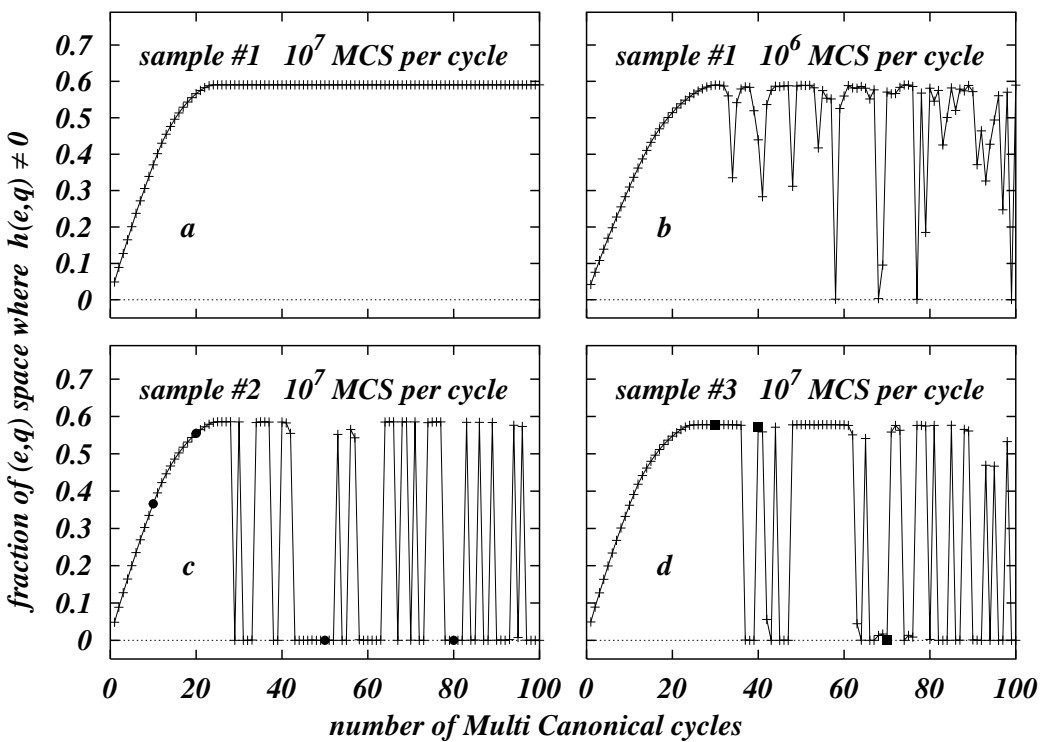

FIG. 3. The fraction of $(e, q)$ space where the histogram $h(e, q)$ is different from zero as a function of the multi-canonical cycle number. Even for a very small system $(L=6)$ strong convergence problems arise. 
Let us focus now specifically on the $3 D$ EA model, and see how the estimated density of states (DoS), $D(e, q)$, converges to the exact one. In particular we are interested in the histogram $h(e, q)$ which counts the number of times, during a multi-canonical cycle, the system is in a macroscopic state $(e, q)$ with energy $e$ and overlap $q$. Thermalization is achieved when $h(e, q)$ is flat and much larger than 1 for all the physically allowed pairs $(e, q)$. Starting from a flat DoS, the region where $h(e, q) \gg 1$ broadens with the number of multi-canonical cycles and eventually reaches the boundaries of the allowed domain, $e \in\left[-e_{0}, e_{0}\right] q \in[-1,1]$, where $-e_{0}$ is the ground-states energy (see the first two snapshots in figure 1 , that we will discuss in better detail later on). In order to describe quantitatively the histogram evolution we plot in figure 3 the fraction of the $(e, q)$ space where $h(e, q) \neq 0$, that is the fraction of macroscopic $(e, q)$ configurations visited by the system during a multi-canonical cycle. We expect this fraction to increase more or less linearly during the first multi-canonical cycles and then to reach a plateau when simulation is thermalized (see figure 3.a, where things look good). For all the $L=4$ samples simulated we have observed this correct behavior. On the contrary for the $L=6$ samples, problems arise. At first, if the number of MCS is not large enough the simulation does not converge at all. In figure 3. b we show the results for the same sample shown in figure 3. a, with the only difference that $10^{6}$ MCS were used instead of $10^{7}$ : here thermalization problems are evident, since in some situations the system simply gets trapped in a very small region of the phase space. In different samples we have found analogous problems also when using $10^{7}$ MCS (see figures 3.c and 3.d). With $10^{6}$ MCS the parallel tempering method is able to thermalize samples up to $L=8$ for temperatures down to $T=0.3$ (for example at the lowest $T$ value the Binder parameter thermalizes in $10^{6} \mathrm{MCS}$ ): the bivariate multi-canonical method does not seem to be very efficient for spin glasses.
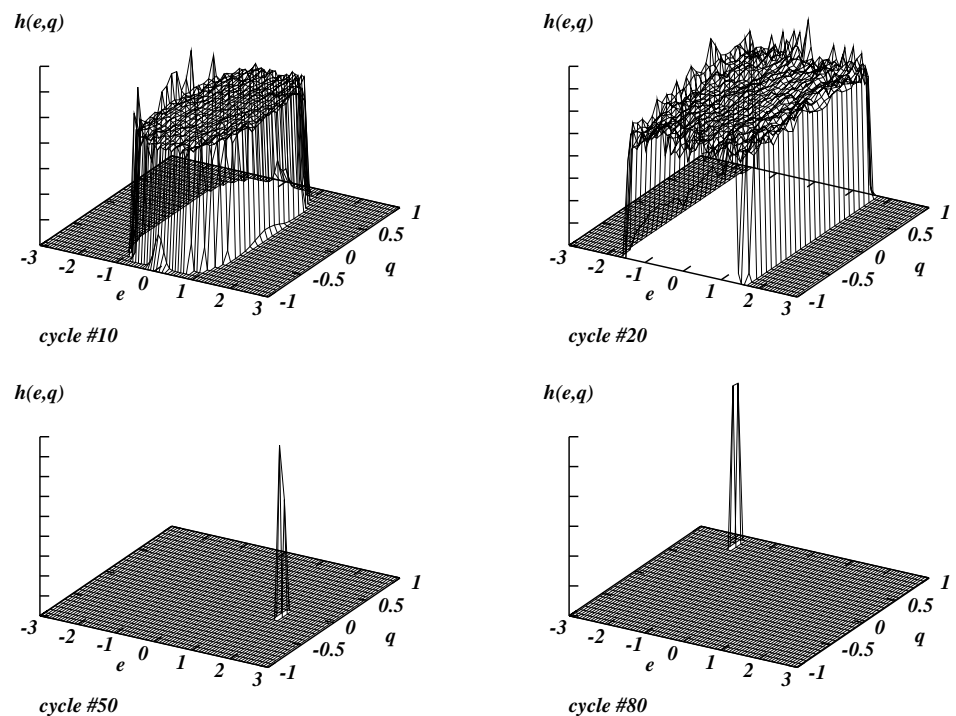

FIG. 4. The evolution of the histogram $h(e, q)$ as a function of multi-canonical cycles (sample \#2 in figure 3).

In figure 4 we show the histogram evolution for sample \#2 (the same used in figure 3.c). The four snapshots correspond to the black dots in figure 3.c and clearly show that the system, after reaching an apparently thermalized state with a flat and broad $h(e, q)$, instead of keeping it for all subsequent times, gets trapped in very small regions of the $(e, q)$ space (the third and fourth snapshots in figure (1).

How can we explain this behavior? During the first multi-canonical cycles the dynamics of the system in the $(e, q)$ space is diffusive in character, while when approaching the boundaries of the $e-q$ plane (especially the energy ones) the system often gets trapped for very long times. The end of the diffusive behavior near to the ground states can be easily explained in terms of accessibility, that is the probability of decreasing the energy when the system is in a $(e, q)$ configuration and it makes a random move to a neighbor configuration. For not too low energies the accessibility is high: in this case a random walk in the configuration space corresponds to a random walk in the $(e, q)$ space, which is a projection of the previous one. On the contrary for energies close to the one of the ground states the accessibility is very low, due to the presence of a large number of higher local minima. For example if the system is at the bottom of a valley in the space of microscopic configurations, in order to further decrease its energy (a little step in the macroscopic $(e, q)$ space) it may need a long time, the time to find a deeper valley. The dynamics turns out to be strongly constrained for energies close to the boundaries.

Having in mind that the dynamics becomes slower and slower close to the energy boundaries, one can easily explain 
the peaks in figure 1 . The system firstly relaxes in a uniform way on a large part of the $(e, q)$ space, the more accessible one. Still many allowed $(e, q)$ values are unvisited (because of the low accessibility), their DoS estimation becomes very small and their corresponding weights, $W(e, q)=1 / D(e, q)$, huge. When the system reaches one of this configurations it can not leave it until the end of the multi-canonical cycle, when $W(e, q)$ will be updated again.

In order to improve the convergence we have also tried to start with a DoS estimated from the one of a thermalized $L=4$ sample. The convergence seems to be faster, however the problems giving rise to the peak structure in the histogram remain unaltered.

Given that the thermalization task appears to be very hard, one should at least try to use all thermalization checks available. For example the one based on the symmetry of the overlap distribution for every sample, $P_{J}(q)$ should always be carefully checked: this analysis is lacking in [1].

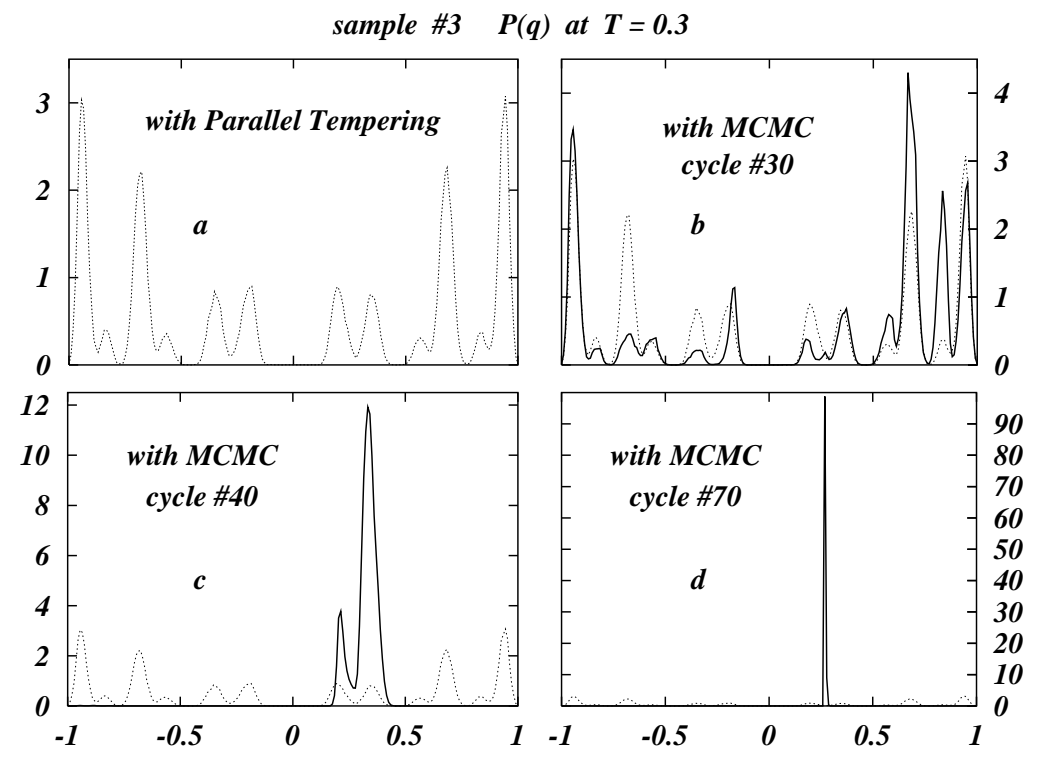

FIG. 5. For a given $L=6$ sample (sample \#3 in figure 3) the $P(q)$ measured with parallel tempering (top left) is symmetric, while it may become much more narrow when a multi-canonical method is employed.

In figure 5 we show the overlap distribution $P_{J}(q)$ for the single $L=6$ sample considered in figure 3.d at a low temperature $T=0.3$ (these data come from a further parallel tempering simulation, pushed to lower $T$ values). In figure 5. a we show the $P(q)$ measured with a parallel tempering simulation. Its very accurate symmetry is a strong evidence of complete thermalization. In the next 3 plots (b,c and d) we show with continuous lines the $P(q)$ measured with the multi-canonical method (the chosen times correspond to the dots in figure 3.d). We always superimpose the thermalized $P(q)$ for comparison. It is clear that, in the best case (see figure 5.b), the multi-canonical method is not able to give results as good as the parallel tempering does: in the worst cases it just gives a completely wrong $P_{J}(q)$, with a single or a double peak. The system may very easily get stuck somewhere, and in these cases the estimated $P(q)$ would look much narrower than the correct one (see figure f.c and figure $\mathbf{5} \cdot \mathrm{d}$ ): measurements taken in such a biased situation hint for a fake evidence in favor of a single peak $P(q)$, and consequently of the droplet scenario.

As a last piece of evidence we consider the samples where the bivariate multi canonical has been well behaved: the scaling of the visited fraction of the $(e, q)$ phase space (for well thermalized samples) reported in figure 6 supports the picture of a diffusion-like evolution of the histogram. The area of support of the histogram grows more or less linearly with the number of multi-canonical cycles (the best exponent estimate is 0.9). Moreover, the time for reaching the plateau (equilibration time) grows with $\tau \propto L^{3.37} \propto N^{1.12}$, which seems to be very close to the theoretical lower bound $(\tau \propto N)$. However this result would hold only if the number of MCS per multi-canonical cycle necessary for a proper thermalization is independent from the system size $N$. As we have already seen this is not true. Indeed, using the same $10^{7}$ MCS per multi-canonical cycle, the fraction of well thermalized samples we have obtained is $100 \%$ for $L=4$, around $40 \%$ for $L=6$ and $0 \%$ for $L=8$. Because the requested number of MCS per multi-canonical cycle grows with $N$ (apparently very fast), our conclusion is that $\tau$ grows much faster than $N$ (simple arguments by Berg [9] suggest at least as $N^{2}$ ).

Concluding, we have seen how difficult it is to bring a bivariate multi-canonical simulation of spin glasses to equilibrium and, consequently, one possible reason of the failure of [1] to thermalize for $L=8$ (we have checked the failure of thermalization with independent parallel tempering simulations). When we say that the simulation is not 
thermalized we mean that we can not use the resulting DoS 2 in order to estimate the observables averages at all the temperature. In particular, as long as the simulation does not visit many times the ground-states, we cannot believe to have enough information on the ground-states structure. However it may perfectly be that, after a certain number of multi-canonical cycles, the estimated DoS gives good averages at higher temperatures, which do not change if new low energy states are reached. We believe this is the case in [1], where data at not too low temperatures are perfectly compatible with the ones obtained in previous work and fit the RSB scenario.

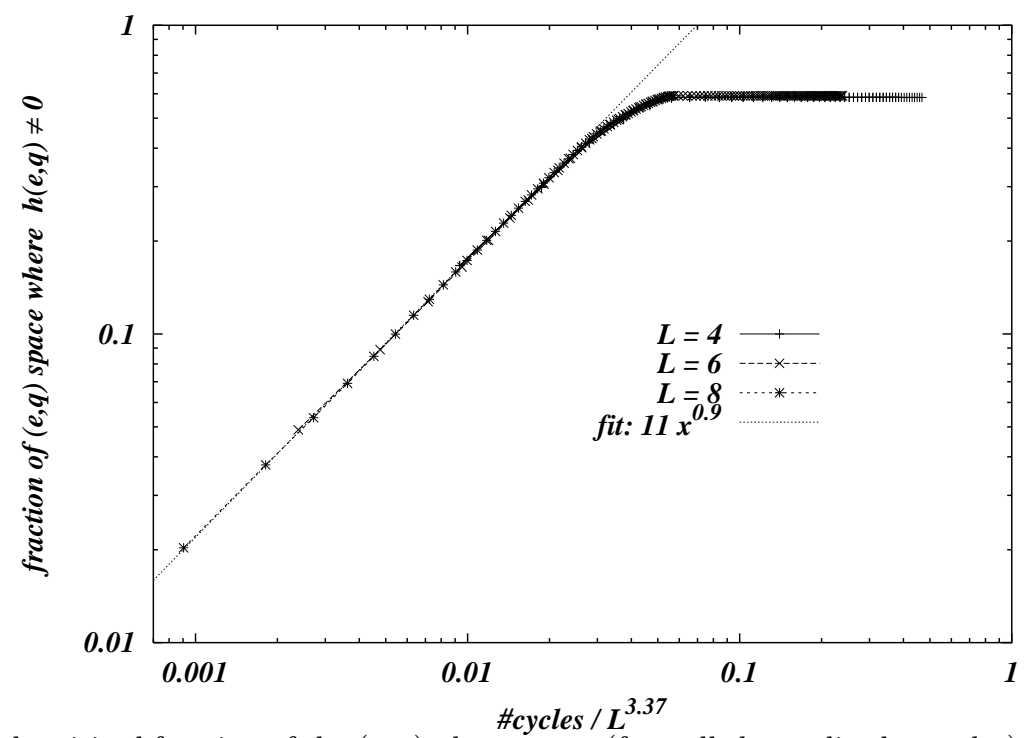

FIG. 6. The scaling of the visited fraction of the $(e, q)$ phase space (for well thermalized samples) shows that the equilibration time must grow with the system size faster than $\tau \propto N^{1.1}$.

We thank N. Hatano for an useful correspondence regarding the bivariate method.

[1] N. Hatano and J.E. Gubernatis, preprint cond-mat/0008115.

[2] H.G. Katzgraber, M. Palassini and A.P. Young, preprint cond-mat/0007113.

[3] G. Parisi, Phys. Rev. Lett. 43, 1754 (1979); J. Phys. A 13, 1101, 1887, L115 (1980); Phys. Rev. Lett. 50, 1946 (1983); M. Mézard, G. Parisi and M.A. Virasoro, Spin Glass Theory and Beyond (World Scientific, Singapore 1987).

[4] K. Hukushima and K. Nemoto, J. Phys. Soc. Japan 65, 1604 (1996). M.C. Tesi, E.J. Janse van Rensburg, E. Orlandini and S.G. Whittington, J. Stat. Phys. 82, 155 (1996).

[5] E. Marinari, Optimized Monte Carlo Methods in Advances in Computer Simulation, edited by J. Kertesz and I. Kondor, Springer-Verlag (1997).

[6] B.A. Berg and T. Neuhaus, Phys. Rev. Lett. 68, 9 (1992).

[7] N. Hatano, private communication.

[8] F. Ritort, Phys. Rev. Lett. 75, 1190 (1995).

[9] B.A. Berg, private communication.

\footnotetext{
${ }^{2}$ Note that the DoS estimation actually used in the measurements in [1] is $D(e, q) h(e, q)$ and so it is strongly affected by non-uniformities in the histogram.
} 